\title{
Health Assistant in Nursing: a Victorian health service pilot
}

\author{
S Davies, C M Keenan and B Redley
}

\begin{abstract}
Objective: Develop and evaluate pilot of a new role to support nursing care delivery in hospital settings.

Design: A naturalistic, three-stage pre-post, multimethod pilot design used data collected from hospital administrative datasets, and surveys and focus groups with staff participants.
\end{abstract}

Setting: Three wards at three hospital sites of a large tertiary health service in Victoria, Australia.

Subjects: Staff performing the new role and registered nurses working on participating wards.

Intervention: Pilot of a new Health Assistant in Nursing (HAN) role.

Main outcome measures: Staff outcomes were work satisfaction and workload of registered nurses; quality outcomes included reported patient falls and medication errors; organisational outcomes included service costs and sick leave.
Results: Work satisfaction and workload of registered nurses remained stable after introducing the new role. The frequency of reported patient falls reduced in two of the three wards. Costing outcomes suggested potential for cost benefits attributed to reduced falls in acute wards.

Conclusions: This pilot identified the new HAN role has capacity to contribute to improved patient quality and safety outcomes without compromising nurse job satisfaction and workload. Potential cost benefits of the new role warrant further consideration in the acute care sector.

Abbreviations: CPO - Constant Patient Observer; CSN - Clinical Support Nurse; HAN - Health Assistant in Nursing. NWSQ - Nursing Workplace Satisfaction.

Key words: Questionnaire; nursing; patient safety; model of care; health assistant; service evaluation.
Sarah Davies Dip Nursing, RN

Project Officer, Nurse Unit Manager

Monash Health

Clayton, Victoria, Australia.

\section{Cathryn Keenan M App Epi, RN}

Project Manager, Deputy Director of Nursing

Nursing \& Midwifery Education and Strategy,

Clayton, Victoria, Australia.

Bernice Redley BN(Hons), Crit Care Cert, PhD, RN Associate Professor of Nursing

Deakin University,

Geelong, Victoria, Australia.

Centre for Nursing Research

Deakin University and Monash Health Partnership,

Burwood, Victoria, Australia.

Correspondence:

bernice.redley@deakin.edu.au

\section{Introduction and background}

Nursing in Australia's health system is facing significant challenges to accommodate future demand and workforce shortages. Forecasts suggest Australia will experience a shortage of approximately 85,000 nurses by 2025 . [1] Rapid population ageing [2] and growing levels of chronic disease, illness acuity and complexity are increasing demands on healthcare. $[3,4]$ Nurses at the forefront of healthcare delivery consistently report concerns that increasing workload demands and limited resource availability impact the quality of care and patient safety. [5] Poor quality, delayed, interrupted, incomplete or missed nursing care $[6,7]$ most often impact patients' personal care needs such as ambulation, hygiene and nutrition [6-9] and is attributed to poor teamwork, resource limitations and nurse workload. [10-12] Suboptimal clinical outcomes and patient dissatisfaction often result. [6] 
In response to Health Workforce Australia's recommendation for changes to the mix of ward staff, [1,3] one Victorian metropolitan and one regional acute health service tested the Health Assistant in Nursing (HAN) during 2009 and the Department of Health and Human Services funded additional health services to undertake similar pilot projects in 2013. The purpose of the HAN role was to support patient centred care under the direct supervision of a registered nurse thereby freeing registered nurses to focus on complex clinical tasks.

This paper reports the process for co-development of the role and evaluation of the pilot to introduce the new HAN role into three different ward types at three different sites of a single large metropolitan health service in Victoria. The role replaced and extended an existing 'Constant Patient Observer' (CPO) role used to supervise patients with high falls risk as the quality and scope of activities performed by CPOs was limited, standards of practice were variable and costs were high. The HAN role was to supplement, not replace, the registered nurse workforce.

\section{Aims and objective}

The aims of the pilot study were to (1) co-develop a new role (HAN) with ward nurses that was expected to provide a scope of activity that would support nursing care delivery in acute and sub-acute wards; and (2) evaluate the impact of the HAN role in terms of staff satisfaction, quality of care and service costs.

The outcomes were expected to inform decisions about the feasibility and ongoing viability of the role and inform a business case for expansion and sustainability.

\section{Methods}

The pilot study utilised a multi-methods pre- and postdescriptive design with data collected from organisational databases, and using surveys and focus groups with staff on participating wards. The setting was a large metropolitan health service in Melbourne, Victoria, Australia. The pilot for the HAN role was introduced at three hospital sites that represented wide variation in service provision:

- Site 1 - An acute 16 bed rapid assessment unit in a tertiary hospital providing treatment of short term acute illnesses for patients with complex underlying disease.

- Site 2 - A 36 bed medical ward in a metropolitan hospital providing treatment of acute and ongoing complex illnesses in elderly patients where high falls risk and delirium and dementia are common.
- Site 3-A 24 bed sub-acute rehabilitation ward that assists elderly patients with a high falls risk to regain independence.

Data collection was guided by a quality evaluation model [14] across three stages. The model included five components: (1) staff costs; (2) quality indicators (falls, medication errors, consumer feedback); (3) staff surveys; (4) focus groups; and (5) ward staff training.

In Stage 1, baseline data were collected from organisational databases and hospital staff. Data were used to describe the existing service, and co-develop a role description and work activity list for the HAN role that was acceptable to nursing staff on the participating wards.

In Stage 2, the role was implemented over six months as follows:

- A Clinical Support Nurse (CSN) was appointed to facilitate the project.

- Seven staff with appropriate qualifications (Certificate III in Health Service Assistance) were recruited using valuesbased interviewing and employed to perform the HAN role.

- A partnership with an external education provider was established to deliver one week of on-site training to the HANs (Certificate III equivalence).

- Nurses on the participating wards were provided with education about the HAN role to manage expectations and enhance understanding of nurse delegation and supervision responsibilities.

- An escalation process to empower staff and capture and respond to staff concerns was established.

In Stage 3, repeat qualitative and quantitative data were collected from staff and organisational databases.

\section{Ethics approval}

Ethics approval was obtained from the participating organisation's Human Research Ethics Committee (ref 14383X).

\section{Data collection and analysis Surveys}

A survey was used to collect data from pilot ward nurses at baseline (Stage 1), and five months after implementation (Stage 3) of the HAN role. In Stage 1, a paper survey was delivered to nurses and subsequently collected by the project CSN. In Stage 3, an email containing an online survey link was sent to nurses and HANs. All survey responses were anonymous. 
The survey included demographic questions, items from the NWSQ [15] and additional fixed response and openended questions. Responses were analysed using the two previously reported themes and six subthemes of the NWSQ: (1) staff work satisfaction (extrinsic factors, intrinsic factors, co-worker factors); and (2) work practices (timeliness and quality of patient care, workload, working with others). [15] Open-ended questions were used to collect data about nurses' perceptions of the HAN role.

\section{Focus groups}

Six focus groups (one pre and one post on each ward) were facilitated by human resources staff; these were conducted in Stages 1 and 3 at each of the three sites. A total of 62 ward nurses participated in Stage 1 focus groups, and 50 in Stage 2. Ward nurses were invited by the CSN to participate and provided written consent. In addition, a workshop attended by 110 nurse managers held in Stage 1 was also used to inform the role development of the HAN. All participants were anonymous. A structured question guide and field notes were used to collect data.

In the Stage 1 focus groups, participants were asked about their:

- Job perception

- Recommendations for HAN tasks

- Support required for success of the pilot

- Their confidence about delegation and supervision.

In Stage 3, the questions relating to job perception were repeated and additional questions were asked concerning implementation processes, resources provided and perceptions of the HAN role impact. Focus group field notes were analysed using thematic data analysis methods common in qualitative descriptive methods.

\section{Organisational quality and costing data}

Quality and costing data were extracted from the organisation's administrative databases including risk reporting, human resources and financial systems. Microsoft Excel was used for statistical analysis of this data.

\section{Results}

Data analyses examined key outcomes of introducing the new co-developed HAN role on the three wards, related to (1) staff and project costs; (2) quality indicators; (3) nursing staff and HAN work satisfaction; and (4) nurse and HAN work practices.

\section{Findings from quantitative analyses Staff and project cost analysis}

Staff costs and cost offsets were calculated for the six-month project period and for the same period in the previous year in each of the participating wards, and then forecast to estimate annual costs. Minimal changes in staff costs occurred in the acute wards and a cost deficit was observed in the sub-acute ward. Sick leave increased during the pilot period across all three wards; however, this was consistent across the organisation and not attributed to the pilot.

Project costs for the HAN pilot were used to estimate costs for subsequent years in order to examine the feasibility of expanding the HAN role. Implementation costs in the first year were forecast to increase in subsequent years when government support was not available.

\section{Quality indicators}

Frequency of medication errors, falls, complaints and compliments were examined for the pilot period and compared with the same period in the previous year.

Incident reports used to examine the frequency of medication administration errors revealed a $31 \%$ (45 to 31) reduction across the three wards during the HAN pilot. Site specific data revealed medication errors reduced only in the two acute wards: 52\% reduction in Ward 1 and $8 \%$ in Ward 2 (see Figure 1).

Data for complaints and compliments received from patients (and their visitors) were small hence pooled across the three wards for analysis. The frequency of complaints reduced by $65 \%(n=11)$ and the number of compliments increased by $560 \%(n=17)$ during the HAN pilot as few compliments were received during the same period in the previous year. Details of compliments related to care quality.

The largest change in quality indicators was observed in the falls data. Overall, the frequency of patient falls decreased by $38 \%$ (144 to 89 ) across the three wards (Figure 2). In the two acute wards, falls reduced by $54 \%$ on Ward 1 and by $49 \%$ on Ward 2. At the same time there was a $13 \%$ reduction in falls across the whole hospital site of Ward 1 and an $11 \%$ increase in falls across the whole hospital site of Ward 2. The frequency of falls in Ward 3 (sub-acute) did not change despite a $20 \%$ reduction in falls at this ward's hospital site for the same time period. Further analysis revealed $81 \%$ of falls on Ward 3 occurred with the HAN was not working. 
Figure 1 Number of Medication Administration Errors in each pilot wards and all pilot wards June - November 2013 vs June - November 2014

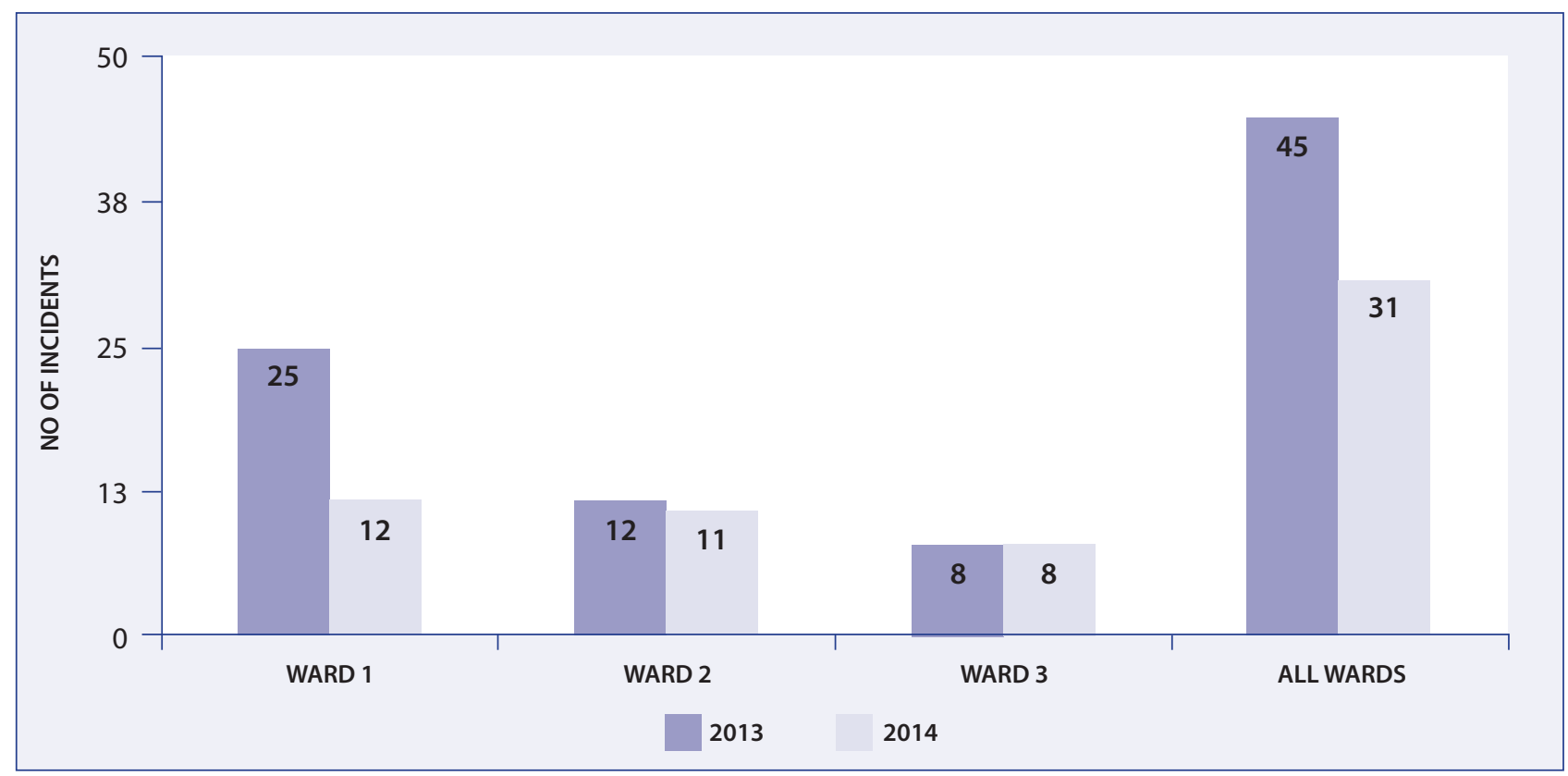

Figure 2: Change in falls frequency in pilot wards and at hospital sites (June - November 2013 vs June - November 2014)

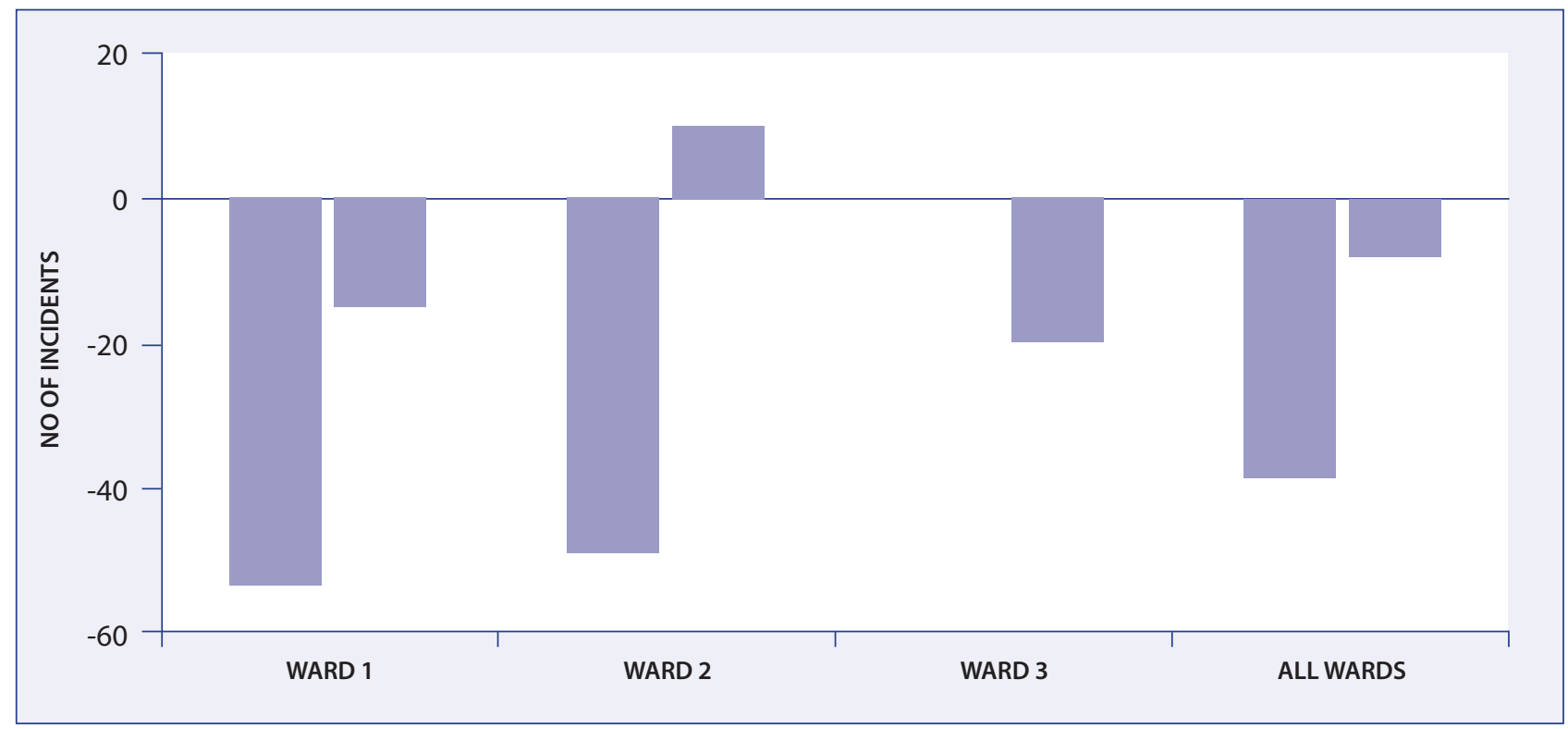

Estimated organisational costs per fall (\$9629) in Australian hospitals [17] were used to project annualised cost savings of falls reduction. Estimated net annual benefits in year one of the project $(\$ 943,318$ (AUS)) and in subsequent years (\$898,760 (AUS)) were observed in acute wards only.

\section{Staff work satisfaction}

The nurse survey response rate was $64 \%(n=67)$ in Stage 1 and $30 \%(n=32)$ in Stage 3. Analyses were limited by the small numbers and inability to match pre and post surveys.
Survey data from Stages 1 and 3 were used to examine the distribution of nurse responses to the three subscales of the NWSQ. $[15,18]$ Differences in subscale scores between Stages 1 and 3 were non-significant for the three subscales, likely due to the sample size lacking power to detect differences; therefore, the response distributions for items in each subscale were examined.

The distribution of responses to the items examining the extrinsic factors affecting work satisfaction [15] revealed 
participant agreement increased in Stage 3 compared with Stage 1 for all items, except items 9 and 12 (see Table 1 for items).

Similarly, agreement with items examining intrinsic factors affecting work satisfaction [15] also increased between Stages 1 and 3 for six of the eight items (Table 1).

Finally, agreement with items related to co-workers [15] also increased between Stages 1 and 3 for three of the four items examined.

\section{Work practices}

In Stages 1 and 3, nurses reported their agreement with items asking about the timeliness and quality of nursing care, workload and working with others (Table 1) using a 5-point Likert scale. For 13 of the 14 items (Table 1) agreement increased in Stage 3 compared with Stage 1. The largest increases in agreement were for items asking about tidying clutter from the work area (item 31) and assisting with patient meals (item 27).
Of the seven items examining nurses' perceptions of their workload (Table 1), the largest increases in agreement were for item 33 (Table 1) indicating benefit of the HAN role, and item 36 (Table 1) suggesting a potential problem in nursing workflow. Agreement increased by $15 \%$ or more between Stage 1 and Stage 3 for four items examining nurses' perceptions of working with others (Table 1).

\section{Perceptions of the HAN role}

In Stage 3, responses from five of the seven HANs to an online survey asking for their perceptions of their role indicated a high level of overall satisfaction with training for the role, transition to the workplace, and stimulating and enjoyable work (all 80\% agreement).

\section{Findings from qualitative analyses}

Sixty-two (59\%) ward nurses participated in focus groups across the three wards in Stage 1 and 50 nurses (48\%) in Stage 3.

Table 1: Stage 1 and Stage 3 responses - Changes to items relating to timeliness and quality of care, workload and working with others

\begin{tabular}{|c|c|c|c|}
\hline \multirow{2}{*}{$\begin{array}{l}\text { ITEMS } \\
\text { TIMELINESS AND QUALITY OF CARE }\end{array}$} & \multicolumn{3}{|c|}{$\begin{array}{c}\text { \% RESPONDING } \\
\text { "ALWAYS" AND “ALMOST ALWAYS" }\end{array}$} \\
\hline & STAGE1 & STAGE 3 & $\%$ CHANGE \\
\hline 19. I believe patient needs are met in a timely manner & 62.7 & 65.7 & $5 \%$ \\
\hline 20. I have adequate time to ensure a safe environment for patient and staff & 72.7 & 78.1 & $7 \%$ \\
\hline 21. I feel that I manage day to day pressures and stress of nursing & 80.6 & 84.4 & $5 \%$ \\
\hline \multicolumn{4}{|c|}{ Please indicate how often you believe these components of care are completed in a timely and satisfactory manner? } \\
\hline 22. Administering medications & 73.1 & 90.6 & $24 \%$ \\
\hline 23. Changing wound dressings & 59.1 & 62.6 & $6 \%$ \\
\hline 24. Performing vital signs & 77.6 & 96.9 & $25 \%$ \\
\hline 25. Patient ADLs: Hygiene- shower/wash & 54.5 & 65.6 & $20 \%$ \\
\hline 26. Grooming (including shaving, hair, teeth/dentures, make up) & 65.6 & 43.8 & $-33 \%$ \\
\hline 27. Patient meals: Sitting down and feeding a patient (where required) & 49.2 & 62.5 & $27 \%$ \\
\hline 28. Patients are assisted to eat their meals whilst the food is still hot & 45.4 & 59.4 & $31 \%$ \\
\hline 29. Talking to patients & 44.8 & 50 & $12 \%$ \\
\hline 30. Answering call bells & 80.6 & 90.7 & $13 \%$ \\
\hline 31. Tidying/cleaning clutter from the work area & 46.3 & 65.7 & $42 \%$ \\
\hline 32. Toileting or attending to toileting needs & 76.1 & 84.4 & $11 \%$ \\
\hline
\end{tabular}


Table 1: Stage 1 and Stage 3 responses - Changes to items relating to timeliness and quality of care, workload and working with others continued

\begin{tabular}{|c|c|c|c|}
\hline ITEMS & \multicolumn{3}{|c|}{$\begin{array}{l}\text { \% RESPONDING } \\
\text { "FULLY AGREE" AND “AGREE" }\end{array}$} \\
\hline WORKLOAD & STAGE1 & STAGE 3 & $\%$ CHANGE \\
\hline $\begin{array}{l}\text { 33. The resources on my ward provide me with the opportunity to apply } \\
\text { my skills and undertake the most important elements of my role }\end{array}$ & 61.2 & 84.4 & $38 \%$ \\
\hline $\begin{array}{l}\text { 34. Workload affects my ability to deliver patients the highest quality } \\
\text { of care }\end{array}$ & 76.2 & 68.7 & $-10 \%$ \\
\hline $\begin{array}{l}\text { 35. Generally for the majority of my shifts I feel I have accomplished } \\
\text { all my daily tasks by the end of shift }\end{array}$ & 74.6 & 77.1 & $3 \%$ \\
\hline $\begin{array}{l}\text { 36. I am regularly interrupted when performing a duty i.e. medication } \\
\text { round, performing a dressing change }\end{array}$ & 53.1 & 84.4 & $59 \%$ \\
\hline 37. On most days I feel that my workload is manageable & 62.6 & 71.9 & $15 \%$ \\
\hline $\begin{array}{l}\text { 38. In comparison to } 3 \text { years ago, I feel that the volume and complexity } \\
\text { of work that I must complete has increased }\end{array}$ & 76.1 & 88.6 & $16 \%$ \\
\hline $\begin{array}{l}\text { 39. In comparison to } 3 \text { years ago, I feel that the standard of care that } \\
\text { I deliver has improved }\end{array}$ & 59.7 & 57.2 & $-4 \%$ \\
\hline WORKING WITH OTHERS & \multicolumn{3}{|c|}{$\begin{array}{c}\text { \% RESPONDING } \\
\text { “FULLY AGREE" AND “AGREE" }\end{array}$} \\
\hline 40. I am confident delegating work to other roles and staff on my ward & 80.6 & 96.9 & $20 \%$ \\
\hline $\begin{array}{l}\text { 41. I am confident providing direct and indirect supervision to other roles } \\
\text { and staff on my ward (when required) }\end{array}$ & 79.1 & 100 & $26 \%$ \\
\hline $\begin{array}{l}\text { 42. I am confident that I know my responsibilities and accountabilities } \\
\text { when delegating and supervising others within my ward }\end{array}$ & 86.6 & 100 & $15 \%$ \\
\hline 43. I feel the staff on my ward work as a team & 80.6 & 96.9 & 20 \\
\hline
\end{tabular}

\section{Stage 1- Nurse work issues}

Nurses reported the best aspects of their role related to:

- Patient care:'Provide good care to patients';

- Good teamwork: 'Working with a great group of people who care about each other'; The opportunity to gain knowledge and skills: 'Learning on the ward, rewarding work'

Worst aspects of their work related to:

- Heavy workloads: 'Heavy work, physically difficult';

- Time constraints: 'Sometimes not having enough time to attend to basic patient care needs due to increased workload/busy ward';

- Pressure from management to increase patient flow: 'Pressure to meet KPIs'.

Work issues related to rostering and equipment were also raised. Nurses' knowledge and confidence about delegation and supervision was variable. Nurses' desire for the HAN role to focus on personal care, feeding, ambulation, toileting and patient supervision also emerged.

\section{Stage 3- Contribution of the HAN role}

In Stage 3, nurses reported good colleagues, teamwork and caring for patients were the best aspects of their work. The worst aspects were lack of management support, pressure for patient flow and high workloads.

Nurses' feedback reflected perceptions that HANs reduced their workloads and freed their time for complex clinical tasks: 'HAN assisted me in giving the best care for my patients by performing duties within their scope, leaves me free to perform duties within my scope'.

Nurses also reported that HANs contributed to:

- Improved patient safety: 'Prevent falls especially for confused patients,' and 'reduced clinical risk' and

- Improved teamwork:'Work alongside other health professionals especially nurses, for best care management for patients.'

Nurses' concerns about the HAN role related to 'Nursing positions being replaced by HANs'. 


\section{Discussion}

\section{Safety and quality improvement}

The findings of this pilot suggest the HAN may contribute to patient safety outcomes by supporting nurses' work. [21] No studies were located in peer reviewed literature to suggest the novel approach to developing and implementing the HAN role reported in this paper may contribute to patient safety; it appears this paper may make a unique contribution to this topic. Previous studies that report assistants in nursing may increase some adverse patient outcomes [17] do not consider the tailored nature of the new HAN roles and the supervision model developed for this pilot that specifically addressed the role, scope-of-practice, and working relationship with registered nurses. [17] The findings suggest that under the right circumstances, the HAN role may contribute to patient safety benefits through reduced falls and medication errors, but this was only evident in the two acute wards where two and four HANs respectively were employed. The employment of only one HAN with limited hours covered may explain the absence of similar improvement in quality indicators on the sub-acute ward, particularly as over $80 \%$ of falls on this ward occurred when the HAN was not on duty. A longer pilot duration and increased hours of cover may improve understanding of HANs'impact on patient safety in sub-acute settings.

\section{Potential for cost benefits}

Since the HAN replaced CPOs on the acute wards there was little additional cost associated with their employment. As CPOs were not usually employed in the subacute ward, the staff costs on this ward were high.

The cost benefits associated with reduced patient falls [18] on acute wards were possibly the most important contribution of the HAN role; an estimated annual benefit of approximately $\$ 898,760$ per annum was projected for the pilot using previously reported costings. [18] This finding suggests the HAN role may offer significant financial benefits in the acute care sector and warrants further investigation.

\section{Nurse work satisfaction}

While changes in nurses' responses to the three sub-scales of the NWSQ between Stages 1 and 3 was non-significant, likely attributable to an inadequate sample size, trends in the data distributions suggest the HAN role may contribute to increased nurse work satisfaction in all subscales. An interesting finding was the mean scores for each of the three NWSQ subscales were higher (by 7.3 to 17.8) in this study population than those reported in previous studies $[15,19,20]$ suggesting the nurse participants had high work satisfaction which may have limited scope for further improvement. Increased nurse work satisfaction was supported by qualitative data that revealed nurses' perceived the HAN role had a positive impact on quality of care and patient outcomes.

\section{HAN role effectiveness}

The introduction of HANs appeared to support nurses to improve their teamwork and workload distribution to maximise the scope-of-practice in each role. This Victorian and Australian context, as no similar literature was located. Feedback from nurses suggested HANs were 'more effective' than the previous CPOs as they could undertake a wider range of patient care activities.

\section{Model of care}

High workloads and poor teamwork well known to affect work effectiveness [21] were expected to be barriers to implementation of the HAN role. Establishing role clarity prior to implementation was fundamental to support effective teamwork. [22] In addition, developing nurses' delegation and supervision skills was seen as key to the HAN's effectiveness. HANs were explicitly made members of the nursing team, attending nurse handovers and reporting directly to a registered nurse on each shift. Delegation and supervision of their work by a registered nurse provided necessary structure and support. This model enabled all team members to focus on their respective role scope and assist each other when needed.

Key factors for successful implementation of the HAN role Underpinning the success of the pilot was engagement, codevelopment and leadership support at all organisational levels. [23,24] Governance using a committee structure facilitated open and transparent two-way communication between management and staff for information-sharing and issue resolution. The dedicated CSN was important to facilitate engagement and support change in local team models of care to create a clearly defined role for the HAN that met expectations and needs. A robust recruitment process and comprehensive education ensured HANs were the 'right fit' and well prepared. Most importantly, ward staff were highly engaged in all stages of the project.

\section{Implications and recommendations}

The findings from the study address the rising nurse workloads and the projected shortage of nurses in Australia by 2025 [1] suggesting a new HAN role, with training and scope-of-practice tailored to the service context, may provide a new health workforce to help mitigate potential for poor quality of care and reduce patient safety risks attributed to high nurse workloads. This pilot study, 
conducted across three sites of one health service, could be replicated elsewhere with different workforce types, as a strategy to progressively address health workforce shortages and improve patient safety.

\section{Limitations}

Due to the high importance of assuring anonymity of participants, demographic data collected was limited, and not matched between the first and second survey; hence it was not known how many nurses participated in both Stages 1 and 3. Similarly, the change from paper (survey 1 ) to online (survey 2) data collection (as a preference of the project team) may have impacted response rates and introduced possibility of response bias. The small sample size limited power to detect differences between pre and post survey data despite evident trends. Limited complaints and compliments data and reliance on self-reporting, which may have been influenced by external factors not considered in this study suggests these findings should be viewed with caution.

\section{Conclusion}

This pilot indicates the HAN role has potential to contribute to improved patient safety and reduced costs in hospital care delivery without compromising nurse satisfaction or workload. The method used for its introduction was key to the success of the pilot. Potential for ongoing cost benefits emerged in the acute care sector where patient falls were reduced. The positive results of this pilot study support proposals to expand the HAN role in the acute sector as a cost effective way to improve patient outcomes and staff satisfaction.

\section{Competing interests}

The authors declare that they have no competing interests.

\section{References}

1. Health Workforce Australia. Nursing Workforce - Retention and Productivity Program. Australian Government; 2014.

2. Australian Bureau of Statistics. Australian demographic statistics. Canberra: ABS;2015

3. Swan W. Australia to 2050: future challenges. Barton, ACT: Department of Treasury; 2010.

4. Schofield DJ, Earnest A. Demographic change and the future demand for public hospital care in Australia, 2005 to 2050. Aust Health Rev. 2006;30(4):507-15.

5. Aiken LH, Sloane DM, Bruyneel L, Van den Heede K, Sermeus W, Consortium RC. Nurses' reports of working conditions and hospital quality of care in 12 countries in Europe. Int J Nurs Stud. 2013; 50(2):143-53.

6. Jones TL, Hamilton P, Murry N. Unfinished nursing care, missed care, and implicitly rationed care: state of the science review. Int J Nurs Stud. 2015;52(6):1121-37.
7. Kalisch BJ. Missed nursing care: a qualitative study. J Nurs Care Qual. 2006;21(4): 306-13.

8. Doherty-King B, Yoon JY, Pecanac K, Brown R, Mahoney J. Frequency and duration of nursing care related to older patient mobility. J Nurs Scholarsh. 2014;46(1):20-7.

9. Kalisch BJ, Landstrom GL, Hinshaw AS. Missed nursing care: a concept analysis. J Adv Nurs. 2009;65(7):1509-17.

10. Aiken LH, Sermeus W, Van den Heede K, Sloane DM, Busse R, McKee $M$, et al. Patient safety, satisfaction, and quality of hospital care: cross sectional surveys of nurses and patients in 12 countries in Europe and the United States. BMJ. 2012;344:e1717.

11. Ausserhofer D, Zander B, Busse R, Schubert M, De Geest S, Rafferty $A M$, et al. Prevalence, patterns and predictors of nursing care left undone in European hospitals: results from the multicountry cross-sectional RN4CAST study. BMJ Qual Saf. 2014; 23(2):126-35.

12. Cho SH, Kim YS, Yeon KN, You SJ, Lee ID. Effects of increasing nurse staffing on missed nursing care. Int Nurs Rev. 2015;62(2):267-74.

13. Health Workforce Australia. Health Workforce 2025 Doctors, Nurses and Midwives. Volume 1. Australian Government; 2012.

14. Donabedian A. Evaluating the quality of medical care. 1966 Milbank Q. 2005;83(4): 691-729.

15. Fairbrother G, Jones A, Rivas K. Development and validation of the Nursing Workplace Satisfaction Questionnaire (NWSQ). Contemp Nurse. 2009;34(1):10-8.

16. Sandelowski M. Whatever happened to qualitative description? Res Nurs Health. 2000;23(4):334-40.

17. Twigg DE, Myers H, Duffield C, Pugh JD, Gelder L, Roche M. The impact of adding assistants in nursing to acute care hospital ward nurse staffing on adverse patient outcomes: An analysis of administrative health data. Int J Nurs Stud. 63:189-200.

18. Haines TP, Hill AM, Hill KD, Brauer SG, Hoffmann T, Etherton-Beer $C$, et al. Cost effectiveness of patient education for the prevention of falls in hospital: economic evaluation from a randomized controlled trial. BMC Med. 2013;11:135.

19. Fairbrother $G$, Jones A, Rivas K. Changing model of nursing care from individual patient allocation to team nursing in the acute inpatient environment. Contemp Nurse. 2010;35(2):202-20.

20. Fairbrother G, Chiarella M, Braithwaite J. Models of care choices in today's nursing workplace: where does team nursing sit? Aust Health Rev. 2015. doi 10.1071/AH14091

21. Parmelee PA, Laszlo MC, Taylor JA. Perceived barriers to effective job performance among nursing assistants in long-term care. J Am Med Dir Assoc. 2009;10(8):559-67.

22. Kalisch B, Lee KH. Staffing and job satisfaction: nurses and nursing assistants. J Nurs Manag. 2014;22(4):465-71.

23. Subirana M, Long A, Greenhalgh J, Firth J. A realist logic model of the links between nurse staffing and the outcomes of nursing. J Res Nurs. 2014;19(1):8-23.

24. Bender M, Connelly CD, Glaser D, Brown C. Clinical nurse leader impact on microsystem care quality. Nurs Res. 2012;61(5):326-32. 